\title{
The Potential for Archaeology Within and Beyond the Habitable Zones (HZ) of the Milky Way
}

\author{
John B. Campbell \\ School of Anthropology, Archaeology \& Sociology, \\ James Cook University PO Box 6811 Cairns, Qld 4870 Australia \\ John.Campbell@jcu.edu.au
}

\begin{abstract}
As archaeology is established on Earth and we are actively exploring the Solar System and beyond, there is the potential to develop a number of forms of exo-archaeology. The archaeology of the things intelligent species do in theory could be practised anywhere, provided one can detect the evidence. Sites are being created by us elsewhere within our star's habitable zone (HZ), namely on the Moon and Mars, and at least molecular traces of human-created probes are being left beyond the $\mathrm{HZ}$ (Venus, Jupiter etc.). The successful detection of extrasolar planets and the possible identification of HZs round other stars raise the possibility for the development of extrasolar archaeology, at least initially by remote sensing techniques. Within the Milky Way the main region to investigate is the galactic habitable zone (GHZ), though there could be archaeological traces of technological behaviours beyond it.
\end{abstract}

\section{Mediocrity and Intelligence}

The Principle of Mediocrity argues for abundant life in the universe, but argues less forcefully for abundant intelligent life (Shostak 1998; Ward \& Brownlee 2000). It took more than 4 billion years ( 4 Gyr) for intelligent life to evolve on Earth, and this was only in the last 2 million years (2 Myr). Although microbial life might be nearly universal, multicellular life might not. This is the basis of the Rare Earth Hypothesis (Ward \& Brownlee 2000), namely that with the special combination of many factors such as plate tectonics, the carbon sink, not too frequent mass extinction events and our supposedly unusual Moon, the Earth has provided evolution with a unique opportunity.

What constitutes intelligence and when did our ancestors first become intelligent? The rise to hominid intelligence began in Africa with the evolutionary branching at about $7 \mathrm{Myr}$ which led to humans (Homo) and the chimpanzee/bonobo clade ( $P a n)$, the antiquity of which is demonstrated by the recent discovery of an early hominid taxon, Sahelanthropus tchadensis (Brunet et al. 2002), dating to 7-6 Myr. The first really major change in hominid brain size comes with our genus Homo at about 2.4-2.1 Myr. The appearance of fully human intelligence starts with the evolution of Homo sapiens sapiens in Africa and its eventual spread elsewhere after about 100000 years ago (100 Kyr). 
The great apes exhibit apparent intelligence, especially bonobos (Pan paniscus) and chimpanzees ( $P$. troglodytes). Recognition of free-living bonobo/chimpanzee cultural behaviours and quite distinct cultural diversity is increasing (McGrew 1992; Mercader, Panger \& Boesch 2002). Great apes make and use tools, but they do not make radios or lasers. However, some have been taught to use computers to communicate and to control their environment themselves (this ability was first demonstrated more than 25 years ago; Rumbaugh 1977).

Exo-archaeology could allow for a wider range of definitions of intelligence, provided the species concerned can alter their environment artificially and provided we can recognise that alteration. We tend to be anthropocentric when it comes to judging intelligence, e.g. the extent to which some of the Cetaceans (dolphins, porpoises and whales) might be intelligent and self-aware is still unresolved (see McShea \& Marino, this volume). If we have trouble with this, how will we be able to recognise intelligence elsewhere in the universe? Then there is the other end of the spectrum. ETIs could have intellects so much in advance of ours that we simply would not be able to understand them or recognise much of what they do (Shostak 1998).

\section{Earth's Archaeological Record}

Till the second half of the $20^{\text {th }}$ century humans did not create sites off world. The archaeological record on Earth stretches back at least 2.4 Myr to the beginnings of our genus in Africa. The oldest recognisable artifacts are stone tools which were made by direct percussion. It took almost $1 \mathrm{Myr}$ for the technology to advance from simple stone tools to partly more advanced tools. The advancements are associated with the evolution of Homo erectus. It (or its allied forms) was also the first human species to spread beyond Africa, to adapt to temperate environments, to capture and use fire, and possibly to make simple watercraft. The origins of anatomically modern humans $(\mathrm{AMH})$ are debated, but the evidence in support of an African origin grows stronger, with AMH originating $120-100 \mathrm{Kyr}$ in Africa and spreading from there. Europe remained the province of Neanderthal people till 40-30 Kyr. Do other intelligent species elsewhere in the Milky Way tolerate the co-existence of more than one intelligent species? We clearly do not. We have gone from multiple species of human and proto-human co-existing in Africa at c. $2 \mathrm{Myr}$ to two species co-existing round $40 \mathrm{Kyr}$ to only one species. But we have replaced species diversity with cultural diversity and a strong ability to invent things independently

\section{The Solar System's habitable zone}

Whether Mars is now completely dead we do not yet know for certain. If Mars has life, we will have some ethical dilemmas. When we colonise Mars, the temptation to change it to something more Earth-like will be very great. This would impact severely on any indigenous life, as well as chemically and physically on any archaeological sites on Mars.

How many and what kinds of sites have we already created beyond the Earth? Sites on the Moon currently include remains of equipment from NASA's Surveyor and Apollo missions, as well as remains of probes sent by the former 
USSR (Luna 2 which landed in 1959 through to Luna 24 in 1976). Before long additional sites will be formed by the European Space Agency (ESA) and Japan. The People's Republic of China has also recently announced the goal of establishing a base on the Moon within the decade.

A site of unique historical significance on the Moon is that of Apollo 11 (Tranquility Base, the first landing site with a human presence). This site should have international recognition and protection, but the procedures for doing that have not yet been established (see O'Leary et al. 2002; Gibson 2001). NASA's second landing site (Apollo 12) is now intended to receive a robotic mission which is being planned by Lockheed Martin to sample remotely some of the site's artefacts and sediments (see Austin et al. 2001). As the first craft to land on the Moon, the former USSR's Luna 2 site should also be considered for protection. The United Nations' Outer Space Treaty (1967) acknowledges the countries which send equipment as the perpetual owners of it, but no-one as the owners or lessees of the sites. A treaty of this sort has no teeth, especially in relation to heritage and its protection, but then it was not designed for this purpose. More recent World Heritage agreements and listings for sites and places on Earth are both inadequate and inappropriate for the Moon or beyond.

Sites on Mars include remains from the former USSR's Mars 3 and Mars 6 (Zak 2002), and the US's Viking missions (soft landings in 1976), Mars Pathfinder (including the Sojourner rover deployed in 1997), Mars Polar Lander (failed soft landing attempt in 1999 with ongoing debate about what happened ${ }^{1}$ ), Mars Climate Observer (crashed in 1999), as well as various bits and pieces from other missions. Artefacts and sites on Mars will be undergoing some decay or abrasion owing to the combined effects of dust storms, hyperaridity, cold temperature variations and possibly even lightning strikes (during dust storms). A ranking in terms of comparative heritage significance would be essential.

Sites on Venus are probably now represented by molecular soup with some trace elements from Earth. We have yet to establish what the corrosion rates actually are and the extent to which they might vary. Whether any sites on Venus should be 'protected' would be quite debatable. However, investigations of just what is left of Magellan since it was sent into the atmosphere in 1994 would be fascinating. Experiments could be trialed with various materials.

Jupiter will not have sites as such, but now scattered within its atmosphere will be chemical traces of the probe launched from Galileo in 1995. Certain asteroids and near-Earth objects (NEO) also have or will have a small amount of human-created space debris or artefacts, such as the NEAR-Shoemaker craft which made a controlled descent on to 433 Eros in 2001 (see Veverka et al. 2001). In the not too distant future, equipment will be landed on a selection of the icy moons of Jupiter and Saturn, such as Europa, which is thought to be a strong candidate for microbial life in its 'sub-glacial' global ocean (see Chyba, this volume; cf. Junge, this volume).

Are there artefacts or sites in the Solar System which have been left behind by other intelligent species? Most of us think not. The "face" and "pyramids" on Mars supposedly recorded by Viking 1 in 1976 have both been shown to vary enormously with the dust storms, and basically not to exist, thanks to detailed

\footnotetext{
${ }^{1} \mathrm{http}: / /$ www.greatdreams.com/lander.htm
} 
imaging done by the Mars Global Surveyor in 1998 and since. The "face" is a mesa with erosion gullies. The "canals" on Mars claimed more than 100 years ago by Percival Lowell have long since been shown not to exist as canals, having been mistranslated from Italian canalli (channels). Water channels are known on Mars, but they are natural and currently presumed dry (Baker 2001).

It would be best not to be overly smug and assume that we do not need to propose protocols for recording artefacts or sites left by other intelligent species either directly or indirectly (e.g. as robotic probes). Some of us may wish to assume that we are totally alone in the Universe, or at least in the Milky Way (e.g. Ward \& Brownlee 2000), but if we treat that assumption as a "fact" then we will not be prepared for dealing with unexpected evidence.

\section{Frequency of Earth-like Planets in other Stellar HZs}

The successful detection of extrasolar planets has now raised the possibility for the development of extrasolar archaeology, at least initially by remote sensing techniques. However, whether Earth-like planets are really rare or common has yet to be determined. NASA's Terrestrial Planet Finder (TPF) and the ESA's Darwin programmes will hopefully increase the chances of sorting this out (see also Beichman, this volume).

Drake Equation (DE) calculations yield a wide range of possibilities for the Milky Way, varying from a few hundred ETI civilizations to about 1 million. Any of these scenarios would now be seen as overly optimistic by Ward \& Brownlee (2000). They have refined the DE and produced what they term the Rare Earth Equation (REE; Ward \& Brownlee 2000). I have combined the two as Ward and Brownlee have left intelligence out. If we are engaged either in SETI or extrasolar archaeology, we need to take intelligence and communicative civilisation lifetimes into account, and at the same time be as fully realistic as possible about the frequency of Earth-analogues.

$$
N=R_{\star} \times f_{p} \times f_{p m} \times n_{e} \times n_{g} \times f_{i} \times f_{c} \times f_{l} \times f_{m} \times f_{j} \times f_{m e} \times f_{I} \times f_{L}
$$

Where:

$R_{\star}=$ birth rate of long-lived stars in the Milky Way

$f_{p}=$ fraction of stars with planets

$f_{p m}=$ fraction of metal-rich planets

$n_{e}=$ planets in stellar HZs

$n_{g}=$ stars in GHZ

$f_{i}=$ fraction of habitable planets where life does evolve

$f_{c}=$ fraction of planets where complex metazoans evolve

$f_{l}=$ percentage of lifetime of a planet which has complex metazoans

$f_{m}=$ fraction of habitable planets with a large moon

$f_{j}=$ fraction of solar-type systems with jovian planets

$f_{m e}=$ fraction of planets with low frequency of mass extinction events

$f_{I}=$ fraction of habitable planets where intelligence evolves

$f_{L}=$ percentage of lifetime of a planet which has communicative civilisations.

Calculations based on this formula certainly yield much lower estimates

than the classical DE, but they do not automatically lead to $\mathrm{N}=1$. 


\section{The Milky Way's Galactic Habitable Zone (GHZ)}

Within the Milky Way the main region to investigate is the GHZ, though there could be archaeological traces beyond it, such as those associated with ETI mining, research activities or perhaps even special astro-engineering projects. Dyson spheres and rings might be constructed round more sorts of objects and systems than originally thought. Infrared searches with refined spectral index discriminants will be required for detecting them (Ekers et al. 2002). Thought has been given previously to looking for traces or emissions which might indicate other kinds of ETI technological activity and even disaster or war. These are listed in the archive of SETI searches (Tarter 2002).

Optical SETI shows considerable promise for increasing the chances of detecting intelligent life, and indeed, for communicating with neighbouring planetary systems, say within 25 light years or within the possible extended lifetime of research teams (see Demorest et al. this volume; McConnell, this volume; Vakoch, this volume). Discovering that other species practise archaeology, astronomy etc. would be almost as incredible as making contact.

\section{Protocols and the Future of Exo-Archaeology}

Should we establish proper protocols for "Space Heritage"? In short, yes. This means that we should determine how we would assess and rank the comparative historical-archaeological significance of sites on the Moon, Mars etc., and then how we would proceed with measures for protection of the more significant sites. For our own off-Earth heritage in the Solar System we should establish and ratify a new United Nations Space Heritage Treaty.

Do we need protocols for "handling" ETI technology which might be present as probes in the Solar System? Again, the answer is yes, though the issue is perhaps less urgent. If we do detect such currently "invisible", possibly polycarbon probes, we will need to think seriously about whether to interfere with them. If such hypothetical probes are parked in stationary orbits, to the best of our knowledge they are not interfering with us.

In the longer run, we should both refine and develop new technologies for remote detection of ETI physical, chemical and biological signatures, especially in the stellar HZs of the Milky Way's GHZ.

\section{References}

Austin, C., Brosseau, P., Castner, M., \& Santana, G. 2001, Visit to the Apollo 12 landing site, www.missionsystems.lockheedmartin.com/exploring/Apollo/index.html

Baker, V. R. 2001, Nature, 412, 228

Brunet, M., et al. 2002, Nature, 418, 145

Ekers, R. D., Cullers, D. K., Billingham, J., \& Scheffer, L.K. 2002, in SETI 2020: A Roadmap for the Search for Extraterrestrial Intelligence, ed. R. D. Ekers, D. K. Cullers, J. Billingham, \& L. K. Scheffer, (Mountain View: SETI Press), 233 
Gibson, R. 2001, Master thesis, (Las Cruces (NM): New Mexico State University)

McGrew, W. C. 1992, Chimpanzee Material Culture Implications for Human Evolution, (Cambridge: Cambridge University Press)

Mercader, J., Panger, M., \& Boesch, C. 2002, Science 296, 1452

O'Leary, B., Hunner, J., Versluis, J., Gibson, R., \& Culp, J. 2002, Lunar Legacy Project, http://spacegrant.nmsu.edu/lunarlegacies/introduction.html

Outer Space Treaty 1967, United Nations Treaty on Principles Governing the Activities of States in the Exploration and Use of Outer Space, Including the Moon and Other Celestial Bodies, New York, United Nations

Rumbaugh, D. M. 1977, Language Learning by a Chimpanzee: The Lana Project, (New York: Academic Press)

Shostak, S. 1998, Sharing the Universe: The Quest for Extraterrestrial Life, (Sydney: Lansdowne)

Tarter, J. 2002, in SETI 2020: A Roadmap for the Search for Extraterrestrial Intelligence, ed. R. D. Ekers, D.K. Cullers, J. Billingham, \& L. K. Scheffer, (Mountain View: SETI Press), 381

Veverka, J., et al. 2001, Nature, 413, 390

Ward, P. D., \& Brownlee, D. 2000, Rare Earth, Why Complex Life Is Uncommon in the Universe, (New York: Springer-Verlag)

Zak, A. 2002, Russia's unmanned missions to Mars, http://www.russianspaceweb.com/spacecraft_planetary_mars.html

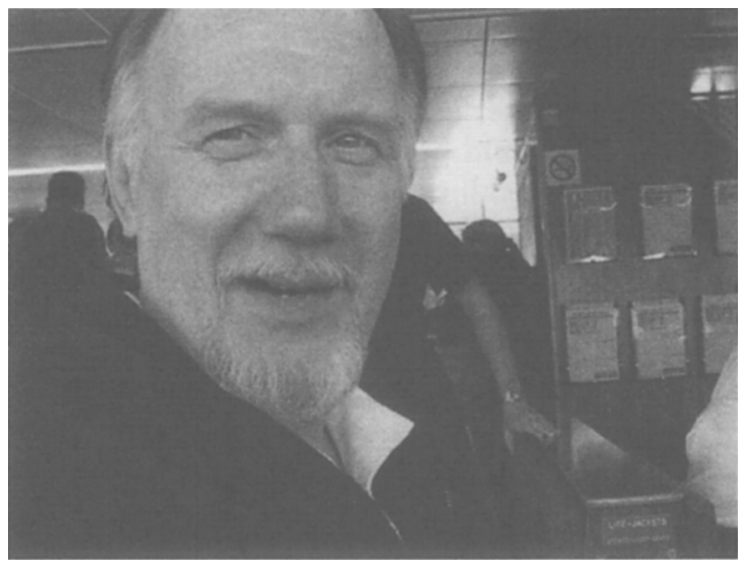

John Campbell (photo: Daniella Scalise) 